\title{
The acquisition of parafoveal word information in reading
}

\author{
HARRY E. BLANCHARD, ALEXANDER POLLATSEK, and KEITH RAYNER \\ University of Massachusetts, Amherst, Massachusetts
}

\begin{abstract}
A somewhat counterintuitive finding has emerged from experiments that restrict the "window" of visual information available on a fixation during reading: fixation duration increases even though there is less information to process on a fixation. The two most likely explanations for this phenomenon are: (1) that the reader extracts abnormal information outside the window and this slows down processing; (2) that a restricted window does not allow a preview of a word before it is fixated, and hence identification of the word is slower when the word subsequently is fixated. In the present experiments, these two alternatives were tested. Conditions in which the size of the window alternated between fixations were compared with conditions in which the size of the window remained constant from fixation to fixation. This manipulation allowed us to separate effects due to restriction of the size of the window on the current fixation from preview benefits (which would be due to restriction of the size of the window on the prior fixation). Two experiments demonstrated clear beneficial effects on fixation duration due to receiving a preview of a word on the fixation prior to when it was fixated. In contrast, restriction of the size of the window had only marginal effects on the fixation on which that restriction occurred. In addition, a subsidiary analysis suggested that the benefit of previewing a word was influenced by its length; for short words, a preview primarily allowed the reader to skip the word more frequently, whereas for longer words, a preview primarily shortened the fixation time on the word when it was later fixated.
\end{abstract}

A very important paradigm for the determination of the details of visual information extraction during reading is the moving window technique developed by McConkie and Rayner (1975; see also Den Buurman, Boersema, \& Gerrissen, 1981; Ikeda \& Saida, 1978; McConkie, Underwood, Zola, \& Wolverton, 1985; Rayner, 1986; Rayner \& Bertera, 1979; Rayner, Inhoff, Morrison, Slowiaczek, \& Bertera, 1981; Rayner, Well, Pollatsek, \& Bertera, 1982; Underwood \& McConkie, 1985). In these experiments, subjects read texts on a cathode-ray tube while their eye movements are monitored, and the displayed text is contingent on where the subject fixates. For example, in a one-word window condition, the word that the subject fixates is presented normally, but the letters in other words are converted to Xs (see Figure 1). By manipulating the size and location of the window and the nature of the material outside the window, one can make inferences about the region from which readers extract information.

This research, which was supported by Grant BNS86-09336 from the National Science Foundation, represents a totally collaborative effort with the order of authors listed alphabetically. H. E. Blanchard is now at AT\&T Laboratories, Holmdel, New Jersey. The work was undertaken while Blanchard was a postdoctoral fellow at the University of Massachusetts. The article was written while Blanchard was at the University of Wisconsin and K. Rayner was at the Netherlands Institute for Advanced Study. Appreciation is expressed to Kevin O'Regan and David Zola for their comments on an earlier version of the manuscript. Portions of the data were reported at the 1988 Midwestern Psychological Association meeting in Chicago. Requests for reprints should be sent to either Alexander Pollatsek or Keith Rayner, Department of Psychology, University of Massachusetts, Amherst, MA, 01003.
The basic finding in such experiments is that the region from which subjects typically extract information is limited, since they read with normal comprehension and speed when they are given a window that extends 4 characters to the left of fixation and 14-15 characters to the right (Rayner \& Pollatsek, 1987). When the window is restricted further, reading slows down but comprehension is unaffected-except in conditions in which the information available is extremely difficult to extract (Rayner \& Bertera, 1979). For example, if the window contains the fixated word and all words to the left, reading speed is about 200 words per minute (wpm), but if the window contains the word to the right of fixation as well, reading speed is increased to about $300 \mathrm{wpm}$. If the window is increased to include two words to the right of fixation, the reading speed is about the same (around $330 \mathrm{wpm}$ ) as when there is no window (Rayner et al., 1982). For convenience, we will refer to these conditions as the one-word window, two-word window, and threeword window conditions, respectively, even though all the words to the left of fixation are visible as well.

Reducing the size of the window affects various measures of reading. When the window gets smaller, the average length of a forward saccade decreases, the number of regressive saccades increases, and the average duration of a fixation increases. Here we wish to focus on the latter phenomenon, because in a way it is paradoxical; the subject fixates longer on a region of text even though there is less information to process. One answer to this apparent paradox is that the subject doesn't know that 
there is less to be processed. In fact, when the material outside the window consists of random letters (and spaces between words are preserved), readers are usually unaware of the window (and the erroneous letters outside it) as long as the fixated word is intact. Thus, in such conditions, the longer fixation durations for smaller windows may be a result of the reader's attempting to extract the usual amount of information but having more difficulty in doing so because some of it doesn't make sense. However, the same phenomenon (longer fixation times with smaller windows) occurs even when all the letters outside the window are changed to Xs. In this situation, the boundary of the window is obvious because of the homogeneity of the field of $X s$, and the reader is aware that the letter information outside the window is of no value.

One explanation for the increase of fixation times with smaller window sizes in the $X$ condition is that the reader's awareness of the window is irrelevant, and that information is still being extracted from outside the window, which disrupts processing. A second explanation is that the effect of the restricted window on fixation time is delayed. According to this second explanation, restricting the window on a fixation (Fixation $N$ ) does not lengthen the duration of Fixation $N$, but instead lengthens the duration of the following fixation (Fixation $N+1$ ).

A plausible mechanism underlying the latter explanation is the parafoveal preview of information. Prior work (Balota, Pollatsek, \& Rayner, 1985; Inhoff, 1987; Lima, 1987; Lima \& Inhoff, 1985; Pollatsek, Rayner, \& Balota, 1986; Rayner, 1975; Rayner, Balota, \& Pollatsek, 1986) has indicated that on one fixation, readers extract letter information that reduces the duration of the following fixation, presumably by allowing the reader to extract partial information from a word to the right of fixation so that it can be identified more efficiently when fixated. While such a parafoveal preview mechanism is almost certainly part of the explanation of why fixation durations are longer with smaller windows, we don't know whether it is the entire explanation or even a major part of the effect.

The current experiments were conducted so that we might be able to understand more precisely the reasons why restricted windows affect reading behavior, especially fixation duration. Two classes of explanation have been indicated above. In one, fixation duration (or some other measure) on Fixation $N$ is affected because the amount of information available on that fixation affects the behavior of the eye. In the other, the fixation duration (or some other measure) on Fixation $N$ is affected because the amount of information available on the prior fixation affects the behavior of the eye. Both explanations are plausible, since it has been demonstrated that events occurring on both the current fixation and the prior fixation can affect the behavior of the eye (Rayner \& Pollatsek, 1981). In the standard window experiment, these two explanations can't be distinguished, because the sizes of the windows on Fixations $N$ and $N+1$ are confounded. The design of the current experiments allowed a test, however, because we varied the size of the window from fixation to fixation.

In particular, in Experiment 1, the size of the window was alternated, so that as the reader moved from word to word, one-word windows and two-word windows were alternately presented. Thus, if fixation duration was primarily affected by the size of the current window, fixations should have been shorter when there was a twoword window on that fixation. In contrast, if fixation duration was primarily affected by the window present on the prior fixation, then fixations should have been shorter when there was a one-word window present on the current fixation (and hence a two-word window was present on the prior fixation). To provide baselines for these alternating conditions, there were two other conditions: one in which there was a constant one-word window, and one in which there was a constant two-word window.

All of the above assumes that control of fixation duration (and other parameters of reading) is completely determined by events that occur on a fixation or soon before, such as on the prior fixation. Rayner and Pollatsek (1981) and McConkie et al. (1985) demonstrated that such immediate control is in fact important in reading. However, there may also be global control of reading behavior. For example, in a more difficult condition, readers may preset themselves to read more cautiously, and in some way adjust the parameters of their system to produce longer fixation times and/or shorter saccades. In other words, with smaller windows, fixation times may be longer and saccades may be shorter at least partly because of global adjustments in the reading process rather than as a reflection of moment-to-moment processing. The design of Experiment 1 did not let us assess these effects, and so for the moment we will not discuss them. However, they were a central focus in Experiment 2.

In addition to permitting an examination of the paradoxical finding that fixation duration increases as windows become smaller, the present experiments also dealt with the nature of parafoveal word processing. In the literature, there is currently some controversy about this issue. Rayner et al. (1986) demonstrated that semantic information is not obtained from unidentified parafoveal words during reading. However, there is some debate about the extent to which partial word information is obtained parafoveally. Two positions have been taken. The first is that partial word information is obtained parafoveally and used to speed the identification of the word on the next fixation. Yet although the results of a number of experiments (cited above, and reviewed in Rayner \& Pollatsek, 1987) support this position, McConkie, Zola, Blanchard, and Wolverton (1982) argued that parafoveal words are either identified completely or not at all. Thus, according to this second position, if a word is identified parafoveally it should be skipped by the next saccade, but if it is not identified, partial information will not be ob- 
tained. The paradigm of alternating the size of the window from one fixation to the next enabled us to differentiate between these two positions.

\section{EXPERIMENT 1}

Our purpose in this study was to examine whether the size of the window primarily affects the behavior of the eye on the current fixation or on the subsequent fixation. So that we might accomplish this, the experimental conditions of interest contained alternating windows, in which the size of the window alternated between one and two words from fixation to fixation. There were also conditions in which window size was held constant at either one or two words, to provide baseline conditions. Lastly, there was a full-line condition, in which the line of text was displayed without restriction; this served as a baseline for the conditions in which the text was restricted.

In addition to varying the size of the window, we also varied the type of information present outside the window. We were interested in determining whether the pattern of results was any different when the letters outside the window were all changed to Xs (and hence the material outside the window clearly did not consist of words), as opposed to when the letters outside the window were changed to visually similar letters so that (as mentioned earlier) the subject was unaware of where meaningful material ended.

\section{Method}

Subjects. Ten adults from the University of Massachusetts community were paid to participate as subjects. They were all experienced in eye-movement experiments, they all had normal uncorrected vision, and they were all native speakers of English.

Materials and Apparatus. One hundred and sixty single-line sentences containing five to eight words were used. The lengths of the sentences extended up to $\mathbf{4 2}$ characters per line. All the sentences were easy to understand, and they contained a variety of syntactic structures.

The sentences were displayed on a Hewlett-Packard 1300A cathode ray tube (CRT), which has a P-31 phosphor that decays to $1 \%$ of maximum brightness after $.25 \mathrm{msec}$. All but the first letters of each sentence were lowercase. A black theater gel covered the CRT to increase the sharpness of the letters. The distance between the subject and the CRT was $46 \mathrm{~cm}$, making $1^{\circ}$ of visual angle equivalent to 3 character positions.

Eye movements were recorded with an SRI Generation III dual Purkinje eyetracker. Movements of the right eye were recorded, although viewing was binocular. Head movements were eliminated by the use of a bitebar. The eyetracker and the CRT were connected to a Hewlett-Packard $2100 \mathrm{~A}$ computer, with the signal from the eyetracker sampled every millisecond and the position of the eye determined about every 3-4 msec. Display changes were completed within 5 msec of a new fixation, and most were probably completed before the fixation was started, since the display change for a new word was initiated when the eye crossed the space between two words. Because the window was defined in terms of the number of words, movements of the eye within a word did not cause a change in the display.

Design and Procedure. A window region was defined inside which the text appeared normal. Outside the window, the text was replaced either by $\mathrm{Xs}$ or by visually similar letters. All text to the left of fixation remained normal; only text to the right of fixation was altered.

Five window-size conditions were used (see Figure 1). In the fullline condition, the entire line was visible on every fixation (this was a normal-reading control condition). In the constant one-word condition, a one-word window was present on each fixation (as well as all the material to the left of fixation). Similarly, in the constant two-word condition, a two-word window was present on each fixation. In the two alternating conditions (12 and 21 ), the window size was determined by the word that was fixated, with the window size alternating from word to word. In the 12 condition, there was a one-word window when odd-numbered words were fixated and a two-word window when even-numbered words were fixated, whereas in the 21 condition, the locations of the one- and two-word windows were reversed. Note that in the alternating conditions, the size of the window was determined by the word that was fixated rather than by the ordinal fixation number. Hence, in the alternating 12 condition, the subject was shown a one-word window when the third word was fixated, regardless of whether the second word was fixated zero, one, or two times.

Crossed with the five window size conditions were two replacement conditions. In one, the text outside the window was replaced with uppercase $X s$, while in the other, each letter was replaced with its most visually similar letter, which was done on the basis of Bouma's (1973) letter-confusability matrix. In both cases, the spaces between words were preserved. The replacement condition was blocked, so that for half the subjects, the first 80 sentences were in the $X$-replacement condition, and for the other half, the first 80 sentences were in the similar-letter replacement condition. Each of the five window sizes occurred in both replacement conditions and was presented in two 8-sentence blocks. The orders of both the blocks and the conditions within blocks were counterbalanced across subjects.

For each subject, the procedure began with the fitting of the bitebar, the calibration of the eyetracker, and the reading of 16 practice sentences. The subject pressed a key to indicate that he or she had finished a sentence, at which time the sentence was removed from the screen. At this point, for some selected sentences, the subject was asked to release the bitebar and recall the sentence. (This was done to ensure that the subjects read for comprehension.) The experimenter then readied the subject and displayed the next sentence. The subjects reported the target sentences with $100 \%$ accuracy.

\section{Results and Discussion}

Three eye-movement measures are presented here: two processing-time measures, mean first fixation duration and mean gaze duration, and the probability of fixating a word. Mean first fixation durations are the means of all first fixation durations for a word, given that the word was fixated. Mean gaze durations are the means of the total fixation time that is spent on a word before the eye moves off to another word; this too is conditional on the word's being fixated at least once. For both measures, data were excluded if the fixation prior to the first fixation on the word was not on the preceding word. This procedure was followed because, as noted before, the window conditions were defined in terms of the word fixated. Therefore, only on the fixations that were preceded by a fixation on the prior word would the window size alternate in the alternating conditions. In addition, data were not included from trials in which there was a disturbance in eyemovement monitoring, such as a track loss; $1 \%$ of the data were lost because of such disturbances. Data anal- 


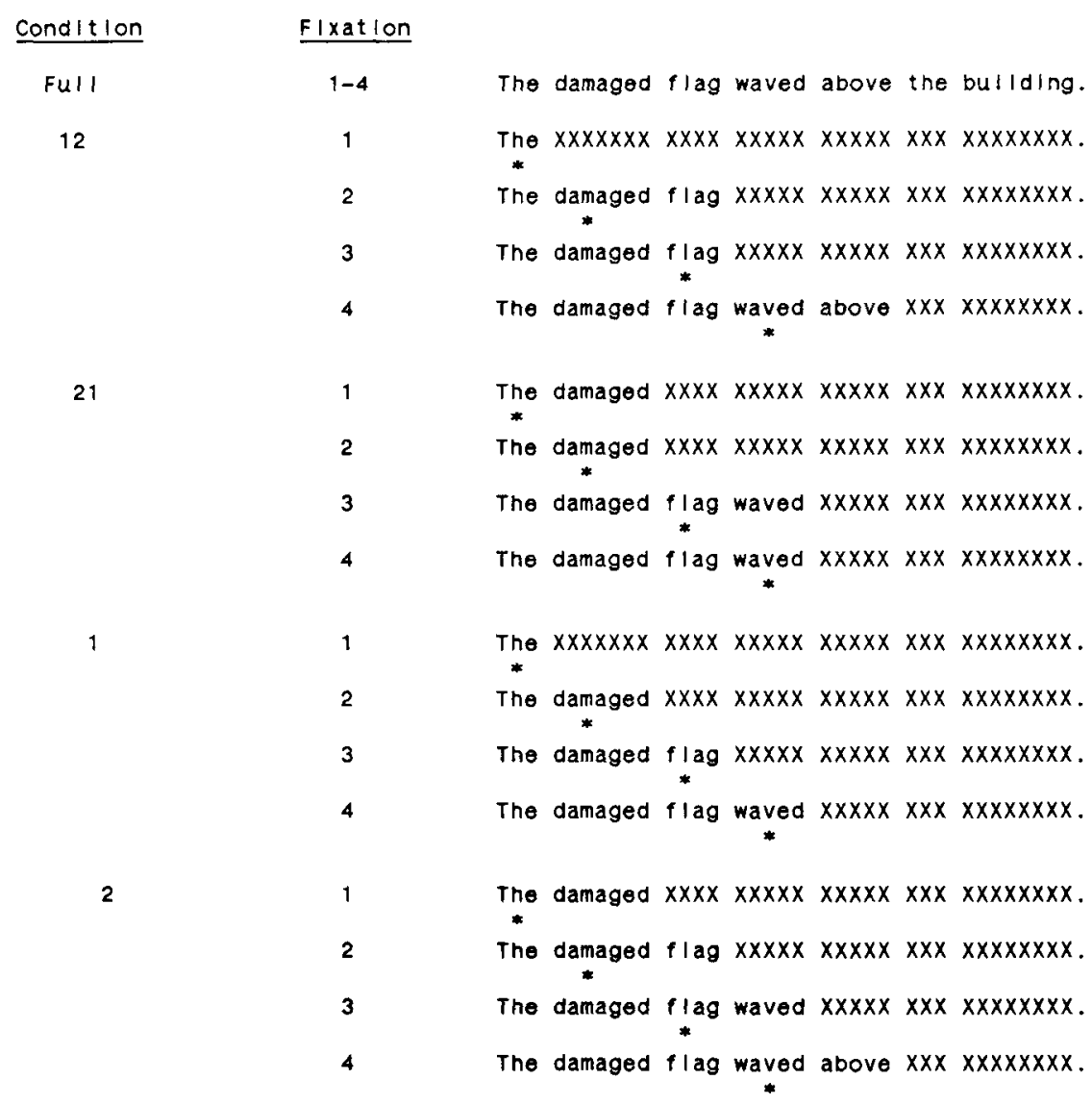

Figure 1. Examples of the experimental conditions in Experiment 1. Four consecutive fixations are shown for each of the experimental conditions (the asterisk marks the location of the fixation).

ysis was also restricted to the fixations on the second through fifth word on a line. The first word was excluded, since the data on it were fairly variable, and data from after the fifth word were excluded, since some sentences had only five words.

The data were preliminarily analyzed with an analysis of variance, which indicated clear effects of the size of the window on first fixation duration $[F(4,36)=22.54$, $p<.001]$, gaze duration $[F(4,36)=23.63, p<.001]$, and probability of fixation $[F(4,36)=4.41, p<.005]$. Neither the replacement condition main effect nor its interaction with window condition approached significance. ${ }^{1}$

Fixation times. Of primary interest, however, were planned contrasts between the various window conditions. For this purpose, the alternating conditions were divided into the occasions on which there was a one-word view and a preview of the fixated word (since the window on the prior fixation was two words) and the occasions on which there was a two-word view but no preview of the fixated word (since the window on the prior fixation was one word). These conditions will be referred to as the alternating one-word-view (A1) and the alternating twoword-view (A2) conditions, respectively. The constant one- and two-word conditions will be denoted $\mathrm{Cl}$ and $\mathrm{C} 2$, respectively.
First we should note that the mean first fixation duration in the full-line condition was $10 \mathrm{msec}$ less than the average from the two preview conditions (A1 and $\mathrm{C} 2$ ) shown in Table $1[t(9)=4.40, p<.005]$, and mean gaze duration was 14 msec shorter $[t(9)=6.85, p<.001]$. This indicates that restricting the window to two words had some effect on the time needed to process individual words.

Of greatest interest, however, is a comparison of preview and no-preview conditions. In the alternating conditions, fixation time was 20 msec less when there was a one-word view than when there was a two-word view, according to the first fixation duration measure $[t(9)=$ $4.61, p<.001]$, and $25 \mathrm{msec}$ less according to the gazeduration measure $[t(9)=3.63, p<.005$; see Table 1$]$. That is, when current window size is pitted against whether or not there was a preview, fixations were shorter for the smaller windows (because there was a preview). Thus, parafoveal preview appears to be the primary reason why fixation durations are shorter in a constant twoword window condition than in a constant one-word condition.

In the alternating conditions, however, the effects of preview and current view are confounded. One can attempt to unconfound these effects, since the four conditions excluding the full-line condition $(\mathrm{C} 1, \mathrm{C} 2, \mathrm{~A} 2$, and 
Table 1

Eye Movement Measures on Words 2-5 in Experiment 1

\begin{tabular}{|c|c|c|c|}
\hline $\begin{array}{l}\text { Window } \\
\text { Condition }\end{array}$ & $X \mathbf{s}$ & $\begin{array}{l}\text { Similar } \\
\text { Letters }\end{array}$ & Mean \\
\hline \multicolumn{4}{|c|}{ First Fixation Duration } \\
\hline $\begin{array}{l}\text { Full } \\
\mathrm{C} 2 \\
\mathrm{~A} 1 \\
\mathrm{~A} 2 \\
\mathrm{C} 1\end{array}$ & $\begin{array}{l}\frac{201}{211} \\
\frac{212}{235} \\
252\end{array}$ & $\begin{array}{l}\frac{209}{219} \\
\frac{216}{232} \\
245\end{array}$ & $\begin{array}{l}\frac{205}{215} \\
\frac{214}{234} \\
249\end{array}$ \\
\hline \multicolumn{4}{|c|}{ Gaze Duration } \\
\hline $\begin{array}{l}\text { Full } \\
\text { C2 } \\
\text { A1 } \\
\text { A2 } \\
\text { C1 }\end{array}$ & $\begin{array}{l}\frac{219}{228} \\
\frac{235}{264} \\
287\end{array}$ & $\begin{array}{l}\frac{229}{245} \\
\frac{242}{263} \\
283\end{array}$ & $\begin{array}{l}\frac{224}{237} \\
\frac{239}{264} \\
285\end{array}$ \\
\hline \multicolumn{4}{|c|}{ Probability of Fixation } \\
\hline $\begin{array}{l}\text { Full } \\
\text { C2 } \\
\text { A2 } \\
\text { A1 } \\
\text { C1 }\end{array}$ & $\begin{array}{l}\frac{.74}{.75} \\
.71 \\
.81 \\
.78\end{array}$ & $\begin{array}{l}\frac{.74}{.72} \\
\frac{.76}{77} \\
.82\end{array}$ & $\begin{array}{l}\frac{.74}{.74} \\
.74 \\
.79 \\
.80\end{array}$ \\
\hline
\end{tabular}

Note- $\mathrm{C} 2=$ constant two-word view. A1 = alternating, one-word view. A2 = alternating, two-word view. $\mathrm{Cl}=$ constant one-word view. Fixation and gaze duration are in milliseconds. Underlined values indicate that the specified word was seen to the right of fixation. Thus, for the fixation-duration data, the word was previewed before it was fixated. For the probability-of-fixation data, the word was visible before it was skipped. The words indicated by nonunderlined values were not visible to the right of fixation.

A1) can be considered as a $2 \times 2$ factorial design containing two levels of current view and two levels of preview. Thus, the best estimate of a preview effect is the average of $\mathrm{C} 1$ and $\mathrm{A} 2$, minus the average of $\mathrm{C} 2$ and $\mathrm{A} 1$. The difference is $27 \mathrm{msec}$ for first fixation duration $[t(9)=5.198$, $p<.001]$, and $36 \mathrm{msec}$ for gaze duration $[t(9)=4.340$, $p<.01]$, indicating a clear effect of preview. The best global estimate of the current view is the average of $\mathrm{Cl}$ and $\mathrm{A} 1$, minus the average of $\mathrm{C} 2$ and $\mathrm{A} 2$. This difference is $7 \mathrm{msec}$ for first fixation duration $[t(9)=2.132$, $p<.10]$, and $12 \mathrm{msec}$ for gaze duration $[t(9)=2.471$, $p<.05]$, suggesting that current view also has some effect. As can be seen from Table 1, however, the currentview effect appears to be different at the two levels of preview. When there was a preview, the current-view effect $(\mathrm{A} 1-\mathrm{C} 2)$ was $-1 \mathrm{msec}$ for first fixation duration and 2 msec for gaze duration, neither of which was significant $(t<1)$, whereas when there was no preview, the current view effect $(\mathrm{C} 1-\mathrm{A} 2)$ was $15 \mathrm{msec}$ for first fixation duration $[t(9)=4.49, p<.005]$, and $21 \mathrm{msec}$ for gaze duration $[t(9)=4.44, p<.005]$.

To summarize, the data indicate that the primary effect on fixation duration is produced by the size of the window on the prior fixation. This suggests that a major determinant of fixation duration is whether or not the fixated word has been previewed on the prior fixation. The data also suggest that there was an effect of current window size on fixation duration as well. However, there are two possible reasons for the latter effect. The first is that fixation times are in fact affected by the current size of the window: fixations are longer when the current window is one word, because the "nonsense" material outside the window slows down processing in some manner. The second is that there is some sort of global control of fixation duration: being confronted with a constant stream of one-word windows causes the reader to slow down and hence causes fixations to lengthen. (The latter explanation is plausible since there were eight consecutive sentences in each window condition.)

Experiment 1 did not allow us to distinguish between these explanations, because current view was confounded with average window size. Since a major thrust of Experiment 2 was to unconfound these two variables, we will defer discussion of this issue until after Experiment 2. However, it is worth mentioning that neither explanation indicates in a natural way why a difference was observed between $\mathrm{C} 1$ and $\mathrm{A} 2$ but not between $\mathrm{A} 1$ and $\mathrm{C} 2$.

Probability of skipping. The other dependent variable of interest was the probability that a word was skipped. There are two aspects of these data that merit discussion. The first is the fact that readers skipped words about $20 \%$ of the time even when the only information obtained in the parafovea before they skipped was the length of the word (e.g., in the one-word window condition). There are two explanations for this phenomenon (they are not mutually exclusive). The first is that the context is sufficient for the reader to identify the next word a certain fraction of the time on the basis of length alone. As a subsequent analysis will show, this is not totally unreasonable, since most of the skipping occurred with short words (three or fewer letters). In the sentences employed, these were usually function words, which are in fact often predictable (Gough, Alford, \& Holley-Wilcox, 1981). The second explanation is that the reader skips the word when it is to the right of fixation but then processes it on the next fixation, when it appears to the left of the fixation point. Prior research (McConkie \& Rayner, 1976; Rayner, Well \& Pollatsek, 1980) had indicated that readers gained no useful information to the left of the fixated word when the right boundary of the window was unrestricted. However, Rayner et al. (1982) presented data indicating that information from the left of the fixated word may be used when the window is restricted to the right.

The second finding of interest is that a word was more likely to be skipped when its letters were present in the parafovea. Overall, words that were visible to the right of fixation were skipped $26 \%$ of the time, while words that were not visible to the right of fixation were skipped $20 \%$ of the time. This difference was significant when the two alternating conditions were compared $[t(9)=$ $3.29, p<.01]$, and clearly neither of the conditions in which the current view consisted of one word differed from each other nor did those in which the current view included two words. In addition, the difference was ap- 
proximately the same in the $X$ conditions as in the similarletter conditions, if one averages over the four relevant conditions ( $\mathrm{C} 1$ and $\mathrm{A} 1$ vs. $\mathrm{C} 2$ and $\mathrm{A} 2$ ).

\section{EXPERIMENT 2}

Experiment 1 clearly indicated that parafoveal preview was the primary determinant of fixation duration. In addition, there appeared to be a small effect of the size of the current window on fixation duration as well. However, the size of the current window was confounded with the global average window size. That is, the average window size in the alternating window conditions was 1.5 words, whereas it was 1 word in the constant one-word condition. Thus, the longer fixation times in the constant oneword window condition could have been due to some global adjustment of fixation duration to the smaller windows in the constant one-word window condition.

In order to unconfound these two possibilities, there were four new control conditions in Experiment 2, in addition to the two alternating-window conditions used in Experiment 1. The latter will be designated as 1212 and 2121 , with the notation 1212 indicating that the window size was 1 when the subject fixated the first word, 2 when the subject fixated the second, 1 when the subject fixated the third, and so forth. (The pattern continues to the end of the sentence, but our notation will indicate only the first four words.) Given the same notation, the four control conditions are 1122(11), 1221(12), 2211(22), and $2112(21)$. In all cases, there is a pattern of two consecutive one-word windows followed by two consecutive twoword windows, and so on. The only difference occurs in how the sequence starts.

The reason for introducing these control conditions was that the average size of the window was 1.5 words in all of them. Thus they were equated to the alternating conditions on average window size, and hence global influences should have caused no difference between these new control conditions and the alternating conditions. In addition, these conditions contained consecutive one-word windows and consecutive two-word windows; thus these conditions contained local points that were identical to the constant one- and two-word window conditions. These control conditions also contained regions that were identical to the alternating-window conditions, so that the alternating conditions and these new control conditions had within them all the conditions of the factorial $2 \times 2$ design, in which the present window size was either one or two words and the prior window size was either one or two words (all having an average window size of 1.5 words for the line). In addition, the fact that the sequence started in one of four places ensured a design that was counterbalanced across serial positions and texts.

Only the $X$-replacement condition was used in Experiment 2 . The $X$-replacement condition was chosen, since it was somewhat easier to interpret (there was no useful information outside the window, instead of information that might have had some benefits and some costs).

\section{Method}

Subjects. Nine subjects participated in this experiment, ? of whom had also participated in Experiment 1.

Apparatus and Procedure. The apparatus and procedure were essentially the same as in Experiment 1. The 144 sentences employed were different from those employed in Experiment 1, although they were similar in length and complexity. The subjects read 16 practice sentences prior to the experimental sentences.

Design. The window regions were defined as in the $X$-replacement conditions of Experiment 1 . That is, the region of normal text extended either from the left of the line to the end of the fixated word (the one-word window) or from the left of the line to the end of the word to the right of the fixated word (the two-word window). As in Experiment 1, in conditions in which the window was changed, the change was defined by the word that was fixated rather than by the ordinal fixation number.

There were nine window conditions in the experiment. The pure one- and two-word conditions and the alternating 1212 and 2121 conditions were exactly as in Experiment 1 . There were four new control conditions, as described above, which had alternating window regions and repeating window regions, designated 1122(11), $1221(12), 2211(22)$, and $2112(21)$, respectively. Lastly, there was a full-line condition as in Experiment 1, in which the subject had an unrestricted view of the text.

Each condition was presented in two blocks of eight sentences each, with the order of conditions and stimulus material counterbalanced across subjects.

\section{Results and Discussion}

Data analyses were done on the mean first fixation duration, mean gaze duration, and probability of fixation, on the second through fifth words of the sentences. Data were excluded by the same procedures as in Experiment 1: data were excluded if the first fixation on a word was not made on the preceding word, and $1 \%$ of the data were also excluded because of track losses. As in Experiment 1 , fixation times were slightly shorter in the full condition than in the other preview conditions. The differences between the full and the other conditions were of about the same magnitude as in Experiment 1 (an 8-msec advantage in first fixation duration, and a 14-msec advantage in gaze duration); however, neither difference in Experiment 2 was significant $[t(8)=1.49$ and $t(8)=$ 1.76 , both $p s>.10]$.

Fixation times. As in Experiment 1, fixations from the alternating (1212 and 2121) conditions were classified according to whether there was a one- or two-word view on the current fixation. Fixations on the 1122, 2211, 1221, and 2112 conditions were classified according to whether the current view contained one or two words and whether the preceding view was the same as or different from the current one. As can be seen in Table 2, the major variable affecting both first fixation duration and gaze duration was the size of the window preceding the present one. Thus, when the preceding window included two words (the underlined conditions in Table 2), the mean first fixation durations ranged between 216 and $229 \mathrm{msec}$, and the mean gaze durations ranged between 253 and $267 \mathrm{msec}$. In contrast, when the prior window contained one word (the nonunderlined conditions in Table 2), the mean first fixation durations ranged between 241 and $250 \mathrm{msec}$, and 
Table 2

Eye-Movement Measures on Words 2-5 in Experiment 2

\begin{tabular}{|c|c|c|c|}
\hline $\begin{array}{l}\text { Window } \\
\text { Condition }\end{array}$ & $\begin{array}{l}\text { First Fixation } \\
\text { Duration }\end{array}$ & $\begin{array}{c}\text { Gaze } \\
\text { Duration }\end{array}$ & $\begin{array}{l}\text { Probability of } \\
\text { Fixation }\end{array}$ \\
\hline Fuil & 218 & 251 & .73 \\
\hline Constant, two-word view & $\overline{227}$ & $\overline{263}$ &.$\overline{75}$ \\
\hline $\begin{array}{l}\text { Same preceding, two-word view } \\
\text { (S2) } 1122,2211,1221,2112\end{array}$ & $\underline{216}$ & $\underline{252}$ & .72 \\
\hline $\begin{array}{l}\text { Different preceding, one-word view } \\
\text { (D1) } 1122,2211,1221,2112 \\
\text { Alternating, one-word view (A1) } \\
\text { Mean of preview conditions }\end{array}$ & $\begin{array}{l}\frac{227}{226} \\
\underline{225}\end{array}$ & $\frac{263}{267}$ & $\frac{.75}{73}$ \\
\hline Alternating, two-word view (A2) & 245 & 294 & .80 \\
\hline $\begin{array}{l}\text { Different preceding, two-word view } \\
\text { (D2) } 1122,2211,1221,2112 \\
\text { Same preceding, one-word view }\end{array}$ & 241 & 284 & .80 \\
\hline (S1) $1122,2211,1221,2112$ & 250 & 287 & .80 \\
\hline Constant, one-word view & 250 & 302 & .80 \\
\hline Mean of no preview conditions & 247 & 292 & .80 \\
\hline
\end{tabular}

Note-Fixation and gaze duration are in milliseconds. Underlined values indicate that word specified received a preview. The words indicated by nonunderlined values did not receive a preview.

the mean gaze durations ranged between 284 and 302 msec.

To get the best estimates of the sizes of the preview (prior window) and current view (current window) effects, two contrasts were computed. (Because differences in global effects might differentiate the pure one- and twoword window conditions from the other conditions, they were excluded from the analyses.) In the equations below, A stands for the alternating conditions, D for the different preview conditions, and $S$ for the same preview conditions (see Table 2); the numbers accompanying the letters indicate the size of the current window.

Preview:

$$
1 / 3[(\mathrm{~A} 1+\mathrm{D} 1+\mathrm{S} 2)-(\mathrm{A} 2+\mathrm{D} 2+\mathrm{S} 1)]
$$

Current view:

$$
.5\{[.5(\mathrm{~A} 1+\mathrm{D} 1)+\mathrm{S} 1]-[.5(\mathrm{~A} 2+\mathrm{D} 2)+\mathrm{S} 2]\}
$$

Hence, the first contrast is merely the average difference between the underlined and nonunderlined conditions in Table 2 (excluding the pure window conditions). For first fixation duration, the value of the first contrast is $23 \mathrm{msec}[t(8)=5.99, p<.001]$; for gaze duration, the value is $28 \mathrm{msec}[t(8)=5.46, p<.001]$. Thus, there was a clear effect of preview. The values of the second contrast assessing the effect of current view were smaller: $9 \mathrm{msec}$ for first fixation duration $[t(8)=2.33, p<.10]$, and $8 \mathrm{msec}$ for gaze duration $[t(8)=0.75]$. There is thus a suggestion that fixations are slightly longer when the window size is reduced, even when the size of the window on the prior fixation is controlled for. Thus, the presence of the $X s$ in the parafovea may slightly slow down the eye for some reason, even when global effects are controlled for.

Word skipping. Similar analyses were conducted on the probability of fixating a word. As can be seen from Table 2 , the reader was $7.0 \%$ less likely to fixate a word when it was visible in the parafovea $[t(8)=3.37$, $p<.01]$. In contrast, the size of the prior window had little effect on skipping; skipping probabilities were only about $2 \%$ less in the D1 and $\mathrm{Al}$ conditions than in the $S 2$ condition $[t(8) \approx 1]$, and they were identical in the $\mathrm{D} 2$ and $\mathrm{A} 2$ conditions and the $\mathrm{S} 1$ condition. Thus, it appears that getting a preview of the fixated word has little influence on the probability of skipping the next one.

Global effects. It is also of some interest to examine global effects that may occur in the pure one- and twoword windows, by using the difference between these conditions and the respective same preceding conditions from the $1122,2211,2112$, and 1221 conditions. First, consider whether readers are slowed down in the pure oneword condition. The pure one-word condition has a mean first fixation duration that is $1 \mathrm{msec}$ less, but a mean gaze duration that is $15 \mathrm{msec}$ more, than in the different preceding condition. (The conditions did not differ in skipping probability.) On the other hand, the pure two-word condition had a mean first fixation duration $11 \mathrm{msec}$ longer, and a mean gaze duration $11 \mathrm{msec}$ longer, than did the comparable same preceding condition, which would be the opposite of what one would expect if there were global effects (i.e., constant presentation of large windows should cause the reader to speed up). Hence, there was little evidence for global effects in Experiment 2.

In sum, the major effect in Experiment 2 (as in Experiment 1) was that fixations were shorter when the word had been previewed in the parafovea. There was a small effect of the size of the current window (that was marginally significant on first fixation duration), suggesting that the presence of $X s$ in the parafovea in place of Word $N+1$ causes fixations to be lengthened slightly. However, there was little indication that readers were slowed down when the global average window size was small (if local viewing conditions are controlled), and there was no suggestion that they were speeded up when the global average window size was large. 
Word-length analysis. The prior means were averaged over words of all lengths. However, it is possible that the preview effects observed are not uniform over words of all lengths. In particular, prior research (Rayner \& McConkie, 1976) has indicated that longer words are not likely to be skipped, whereas shorter words are quite likely to be skipped. Thus, it is possible that for short words, all of the benefit of a preview takes the form of a greater probability of skipping that word, whereas for longer words, most of the benefit may take the form of shorter fixations on that word when it is later fixated. If so, then one would have to be wary of drawing general conclusions about the effects on all words, and one would have to specify the length of the words involved.

In order to examine how the length of a word affects how much benefit the word receives from a preview, data from Experiments 1 and 2 were combined, since a large data sample was needed to get sufficient data for various word lengths. This analysis was performed only on the data from the alternating conditions, in which there were $X$ s outside the window. Words were classified into three length groups: words that were 1-3, 4-5, or 6-10 letters in length. Table 3 presents the data from the combined experiments for processing-time measures.

As can be seen, there was a trend in both fixationduration measures for the preview effect to be bigger for longer words. For first fixation duration, the preview effect increased from $2 \mathrm{msec}$ for the smallest words to $25 \mathrm{msec}$ for the longest words. Similarly, for gaze duration, the preview effect increased from $11 \mathrm{msec}$ to $39 \mathrm{msec}$. However, both of the interactions of word length $\times$ window were only marginally significant $[F(2,22)=$ $3.030, p=.07$, for first fixation duration, and $F(2,22)$ $=3.295, p=.06$, for gaze duration]. Simple effects showed significant window effects for the longer length words on both measures (all $p s<.01$ ), and clearly no significant window effect for the one- to three-letter words. A more detailed examination of the data indicated that the lack of significance in the interactions was due

Table 3

Combined Data from the Alternating Conditions of Experiments 1 and 2

\begin{tabular}{lccc}
\hline $\begin{array}{c}\text { Word } \\
\text { Length }\end{array}$ & $\begin{array}{c}\text { One-Word View } \\
\text { (Preview) }\end{array}$ & $\begin{array}{c}\text { Two-Word View } \\
\text { (No Preview) }\end{array}$ & Difference \\
\hline & \multicolumn{4}{c}{ First Fixation Duration } \\
1-3 letters & 214 & 216 & \\
4-6 letters & 218 & 242 & 24 \\
6-10 letters & 230 & 255 & 25 \\
& \multicolumn{2}{c}{ Gaze Duration } \\
1-3 letters & 219 & 230 & \\
4-6 letters & 238 & 265 & 11 \\
6-10 letters & 284 & 323 & 27 \\
& \multicolumn{4}{c}{ Probability of Fixation } \\
1-3 letters & .44 & .57 & 39 \\
4-6 letters & .80 & .90 & .13 \\
6-10 letters & .94 & .96 & .10 \\
\hline
\end{tabular}

Note-Fixation and gaze duration are in milliseconds. to the window effects' being much more variable for the smaller words; this was due, at least in part, to there being many fewer observations for these words, since they were skipped much more frequently (see Table 3 ). In addition, we should point out that the length analysis indicated that longer words were fixated for longer times than were shorter words $[F(2,22)=6.750, p<.01$, for first fixation duration, and $F(2,22)=24.43, p<.001$, for gaze duration].

For the probability of fixation, there was the opposite pattern. First, there was a clear effect of length, with the shorter words being skipped almost half the time and the longer words being skipped only $5 \%$ of the time $[F(2,22)$ $=171.1, p<.001]$. In addition, the effect of the window was more pronounced for the smaller word sizes, with a preview increasing word skipping by $13 \%, 10 \%$, and $2 \%$ for the short, medium, and longer words, respectively. However, the interaction of length $x$ window was again only marginally significant $[F(2,22)=3.368, p=$ .053].

The data thus suggest the hypothesis that a parafoveal preview of a word has somewhat different effects for shorter than for longer words. For shorter words (1-3 letters), a preview increases the probability that the word will be skipped, but the preview has no clear effect on the time that the word is fixated. In contrast, for long words (6-10 letters), a preview shortens the time the word is fixated, but has only a small effect on the probability that the word will be skipped, since words of this length are almost always fixated. For the medium-sized words (3-5 letters), a preview affords both benefits: the word is skipped more if previewed, and if not skipped, the word is fixated for a shorter period of time.

\section{GENERAL DISCUSSION}

The major finding in the present study was that when the current window size and the window size on the prior fixation were unconfounded, the effects of window size on the time of fixation on a word (measured by either the first fixation duration or the gaze duration) were virtually all due to the size of the window on the prior fixation.

In each experiment, however, there was a suggestion of an effect due to window size beyond the preview effect. In Experiment 1, the difference between the oneword- and two-word-view conditions was $7 \mathrm{msec}$ for first fixation duration and $11 \mathrm{msec}$ for gaze duration, with the latter difference being significant. In Experiment 2, the analogous differences were 9 and $8 \mathrm{msec}$ for first fixation duration and gaze duration, respectively, with the former being marginally significant. Thus, having nonsense material in the parafovea appears to have a small effect on the current fixation duration. The data of Experiment 2 , however, indicate that global control has little effect on fixation time, for there was little difference between the constant-window conditions and those fixations in the alternating conditions that were equated for current and immediately prior window size. 
In addition, window size appears to affect the probability of fixation almost exclusively by allowing readers to skip words more when they are seen to the right of the fixated word. Moreover, the data indicate that readers were skipping words that they had neither seen to the right of fixation nor fixated. From the present data, it is uncertain whether they were able to identify these words on the basis of context and word length, or whether they identified these words when they were to the left of the fixated word, or both. Prior work (Rayner et al., 1982) has indicated that with window restrictions to the right of fixation, readers seem to extract some information to the left of the fixated word (whereas with no window restrictions to the right of fixation, readers use no information to the left of the fixated word).

As in prior experiments (e.g., Rayner et al., 1982), reading was not quite as fast in a two-word-window condition as it is with normal text, indicating that information from more than two words was being extracted, at least on some fixations. However, since the difference between the full-line and two-word-window conditions is small, it appears that reading primarily involves the extraction of information from the fixated word and the one to the right, and the integration of those words into higher order syntactic and discourse structures.

The present experiments indicate that the time of fixation on a word is strongly influenced by the word's length. In natural discourse, of course, word length is highly confounded with the frequency of the word in the language and with the predictability of the word in the sentence (which may be only partly due to word frequency). However, in other experiments (e.g., Inhoff \& Rayner, 1986; Rayner \& Duffy, 1986), it has been demonstrated that fixation time is affected by word frequency, even when word length and the predictability of the word are controlled for. In any event, the fact that length and frequency have effects on fixation time indicates that the time of fixation on a word is influenced by the time spent accessing the fixated word. More generally, our data argue for a close relationship between the visual information present on the page on the current as well as the prior fixation and the behavior of the eye. The effects observed that were due to global variables, such as the average window size, were marginal and not consistent across conditions. This is methodologically important, since it indicates that the effects of window manipulations are quite directly related to the information being processed at the moment rather than to more general, and probably less interesting, global strategies devised to deal with window conditions.

There are two plausible reasons why restricting the current window might increase fixation time. One is that when the subject sees "nonsense" to the right of fixation, the rightward eye movement is inhibited. On this view, one would expect a current-view effect when there are $X s$ outside the window, since the reader is consciously aware of them, but not when there are similar letters outside the window, since the reader is rarely if ever con- sciously aware of them. The data from Experiment 1 indicate that this hypothesis may account for part of the current-view effect, since the current-view effect was somewhat larger when there were $X s$ outside the window rather than similar letters ( 9 vs. 6 msec for first fixation duration and 15 vs. 9 msec for gaze duration). The other possibility is that the processing of parafoveal letters that allow lexical access of Word $N+1$ to proceed smoothly (as is the case when there is a two-word window) allows an eye movement to be programmed sooner. At present, however, there are no data supporting this hypothesis. For example, the time of fixation on Word $N$ is unaffected by the frequency of Word $N+1$ (Carpenter \& Just, 1983; Rayner \& Duffy, 1986), provided that Word $N+1$ is not skipped.

While the effect of current view on fixation time is marginal and not well understood, the preview effect is quite robust, and, as indicated in the introduction, several lines of evidence indicate that lexical access of a word is speeded by a parafoveal preview of it. One possible mechanism for this facilitation is that the letters of the parafoveal word partially activate a neighborhood of lexical entries, including the entry of the word actually visible, so that the resulting excitation speeds lexical access when the word is actually fixated. Another possibility is that abstract letters are identified parafoveally, which facilitates lexical access on the subsequent fixation (Rayner \& Pollatsek, 1989). In either case, the data indicate that in normal reading, many words are processed on more than one fixation. This conclusion has several methodological consequences.

The first is that although the time of fixation on a word is a sensitive index of lexical access time, fixation time (either first fixation duration or gaze duration) as a direct measure of word-identification time must be interpreted with caution, since part of the time spent processing many words occurs before they are fixated. (Of course, all the time spent processing words that are skipped is other than the time when they are fixated.) The second point is that methods which expose only one word at a time (as in our one-word-window conditions) slow down reading. In fact, in experiments in which the text is advanced word by word with manual keypresses rather than eye movements, reading is slowed down considerably (see Aaronson \& Ferres, 1984; Just, Carpenter, \& Woolley, 1982). It may be hard to generalize from such conditions to normal reading merely because the process is slowed down (Rayner \& Frazier, 1987). It is possible, however, that the elimination of parafoveal previews may alter the reading process qualitatively as well as quantitatively. For example, when a word is not visible in the parafovea, the effects of prose context on lexical access may be highly exaggerated. In normal reading, it seems likely that the gap between the time when the meaning of Word $N$ is accessed and Word $N+1$ is fixated is occupied (at least in part) by the extraction of the visual information from Word $N+1$ in the parafovea. In contrast, when Word $N+1$ is not visible, the reader may spend much of the time between the 
identification of Word $N$ and fixating Word $N+1$ in trying to use context to anticipate Word $N+1$. Such considerations suggest that great caution should be exercised when generalizing from conditions in which reading is done without parafoveal previews to normal reading.

\section{REFERENCES}

Aaronson, D., \& FERres, S. (1984). The word-by-word reading paradigm: An experimental and theoretical approach. In D. Kieras \& M. Just (Eds.), New methods in reading comprehension research (pp. 31-68). Hillsdale, NJ: Erlbaum.

Balota, D. A., Pollatsek, A., \& Rayner, K. (1985). The interaction of contextual constraints and parafoveal visual information in reading. Cognitive Psychology, 17, 364-390.

BoumA, H. (1973). Visual interference in the parafoveal recognition of initial and final letters of words. Visual Research, 13, 767-782.

CARPenter, P. A., \& Just, M. A. (1983). What your eyes do while your mind is reading. In $\mathrm{K}$. Rayner (Ed.), Eye movements in reading: Perceptual and language processes (pp. 275-307). New York: Academic Press.

Den Buurman, R., Boersema, T., \& Gerrissen, J. F. (1981). Eye movements and the perceptual span in reading. Reading Research Quarterly, 16, 227-235.

Gough, P. B., Alford, J. A., JR., \& Holley-Wilcox, P. (1981). Words and contexts. In O. L. Tzeng \& H. Singer (Eds.), Perception of print: Reading research in experimental psychology (pp. 85-102). Hillsdale, NJ: Erlbaum.

IKEDA, M., \& SAIDA, S. (1978). Span of recognition in reading. Vision Research, 18, 83-88.

INHOFF, A. W. (1987). Parafoveal word perception during eye fixations in reading: Effects of visual salience and word structure. In M. Coltheart (Ed.), Attention and performance: 12. The psychology of reading (pp. 403-418). London: Erlbaum.

INHOFF, A. W., \& RAYNER, K. (1986). Parafoveal word processing during eye fixations in reading: Effects of word frequency. Perception \& Psychophysics, 40, 431-439.

Just, M. A., Carpenter, P. A., \& Woolley, J. D. (1982). Paradigms and processes in reading comprehension. Joumal of Experimental Psychology: General, 111, 228-238.

LimA, S. D. (1987). Morphological analysis in sentence reading. Journal of Memory \& Language, 26, 84-99.

LimA, S. D., \& INHOFF, A. W. (1985). Lexical access during eye fixations in reading: Effects of word-initial letter sequence. Journal of Experimental Psychology: Human Perception \& Performance, 11, 272-285.

McConkIE, G. W., \& RAYner, K. (1975). The span of the effective stimulus during a fixation in reading. Perception \& Psychophysics, 17, 578-586.

MCConkIe, G. W., \& RAYNer, K. (1976). Asymmetry of the perceptual span in reading. Bulletin of the Psychonomic Society, 8, 365-368.

MCConkIE, G. W., Underwood, N. R., Zola, D., \& Wolverton, G. S. (1985). Some temporal characteristics of processing during reading. Journal of Experimental Psychology: Human Perception \& Performance, 11, 168-186.

McConkie, G. W., Zola, D., Blanchard, H. E., \& Wolverton, G. S. (1982). Perceiving words during reading: Lack of facilitation from prior peripheral exposure. Perception \& Psychophysics, 32, 271-281.

Pollatsek, A., Rayner, K., Balota, D. A. (1986). Inferences about eye movement control from the perceptual span in reading. Perception \& Psychophysics, 40, 123-130.
RAYNER, K. (1975). The perceptual span and peripheral cues in reading. Cognitive Psychology, 7, 65-81.

RAYNER, K. (1986). Eye movements and the perceptual span in beginning and skilled readers. Joumal of Experimental Child Psychology, 41, 211-236.

Rayner, K., Balota, D. A., Pollatsek, A. (1986). Against parafoveal semantic preprocessing during eye fixations in reading. Canadian Joumal of Psychology, 40, 473-483.

RAYNER, K., BERTERA, J. H. (1979). Reading without a fovea. Science, 206, 468-469.

RAYNER, K., \& DUFFY, S. A. (1986). Lexical complexity and fixation times in reading: Effects of word frequency, verb complexity, and lexical ambiguity. Memory \& Cognition, 14, 191-201.

Rayner, K., \& Frazier, L. (1987). Parsing temporarily ambiguous complements. Quarterly Joumal of Experimental Psychology, 39A, 657-673.

Rayner, K., Inhoff, A. W., Morrison, R. E., Slowiaczek, M. L., \& BERTERA, J. H. (1981). Masking of foveal and parafoveal vision during eye fixations in reading. Journal of Experimental Psychology: Human Perception \& Performance, 7, 167-179.

RAYNER, K., \& MCCoNKIE, G. W. (1976). What guides a reader's eye movements? Vision Research, 16, 829-837.

Rayner, K., \& Pollatsek, A. (1981). Eye movement control during reading: Evidence for direct control. Quarterly Joumal of Experimental Psychology, 33A, 351-373.

Rayner, K., PollatseK, A. (1987). Eye movements in reading: A tutorial review. In M. Coltheart (Ed.), Attention and performance: 12. The psychology of reading (pp. 327-362). London: Erlbaum.

Rayner, K., \& POllatsek, A. (1989). The psychology of reading. Englewood Cliffs, NJ: Prentice-Hall.

Rayner, K., Well, A. D., \& Pollatsek, A. (1980). Asymmetry of the effective visual field in reading. Perception \& Psychophysics, 27, 537-544.

Rayner, K., Well, A. D., Pollatsek, A. \& Bertera, J. H. (1982). The availability of useful information to the right of fixation in reading. Perception \& Psychophysics, 31, 537-550.

Underwood, N. R., \& McConkIE, G. W. (1985). Perceptual span for letter distinctions during reading. Reading Research Quarterly, 20, 153-162.

\section{NOTE}

1. In two prior experiments (McConkie \& Rayner, 1975; Rayner et al., 1982 ) an advantage was found for $X$-replacement over similar-letterreplacement conditions for overall reading rate, average fixation duration, and average saccade length. However, the effects on average fixation duration were relatively modest (differences averaging about $10 \mathrm{msec}$ ), and the differences were more pronounced for larger windows than for smaller windows. (Why the exact nature of the material outside the window should be more important when it is further from fixation is not clear.) In contrast, Inhoff and Rayner (1986) obtained results that were the same as those in the present experiment: there was no difference between the two replacement conditions. The cause of the inconsistency is not clear. One difference is that different measures were employed: first fixation duration and gaze duration in Inhoff and Rayner and in the present experiment, and average fixation duration in McConkie and Rayner (1975) and Rayner et al. (1982).

(Manuscript received July 25, 1988; revision accepted for publication January 4 , 1989.) 